\title{
Modifiye Hummers Yöntemi ile Elde Edilen Grafen Oksit Sentezleri İçin: Kısım 4, Raman Spektroskopisi Analizi
}

\author{
Ömer Laçin ${ }^{1 *}$, Bünyamin Dönmez ${ }^{1}$ \\ ${ }^{1 *}$ Atatürk Üniversitesi, Mühendislik Fafültesi, Kimya Mühendisliği Bölümü, Erzurum, Türkiye (ORCID ID 0000-0002-5276-3056), olacin@atauni.edu.tr \\ ${ }^{1}$ Atatürk Üniversitesi, Mühendislik Fafültesi, Kimya Mühendisliği Bölümü, Erzurum, Türkiye (ORCID ID 0000-0002-7680-0755), bdonmez@atauni.edu.tr
}

(1st International Conference on Applied Engineering and Natural Sciences ICAENS 2021, November 1-3, 2021)

(DOI: $10.31590 /$ ejosat.1012390)

ATIF/REFERENCE: Laçin, O. \& Dönmez, B. (2021). Modifiye Hummers Yöntemi ile Elde Edilen Grafen Oksit Sentezleri İçin: Kısım 1, Raman Spektroskopisi Analizi. Avrupa Bilim ve Teknoloji Dergisi, (27), 993-997.

$\ddot{O} \mathbf{z}$

Bu çalışmada, Raman Spektroskopisi analizi ile Hummers yöntemindeki sodyum nitrat konsantrasyonunun değiş̧irilmesiyle elde edilen sentezler sonucunda grafen okside dönüşümü ve yapısal özelliklerinin değişimi incelenmiştir. Grafitin Raman spektrumunda; 1573,7 $\mathrm{cm}^{-1}$ de G (\%32,3 pik alanlı) ve 2692,3 $\mathrm{cm}^{-1}$ de 2D (\%54,1 pik alanl1) karakteristik bantları görülmüştür. Grafen oksit sentezlerinin Raman spektrumlarında ise sirasıyla; 1582,1 ve $1591,2 \mathrm{~cm}^{-1}$ 'de G bandı ( \%23 pik alanlı) ve 1352,3 ve 1355,8 $\mathrm{cm}^{-1}$ 'de bir D bandı ( \%51 pik alanlı) olmak üzere iki belirgin pik gözlenmiştir. Buna ilaveten sentez numunelerinde $\sim 2928-2947 \mathrm{~cm}^{-1}$ ' $d e$ yeni bir D+D' bandı ( $\sim 12$ pik alanlı) ortaya çıkmış ve grafitte $3228 \mathrm{~cm}^{-1}$ 'deki 2D' bandı ise kaybolmuştur. Kimyasal oksidasyondan sonra, grafitteki D bandı pik alanının \%10,8 iken grafen oksit sentezlerinde $\sim \% 51$ 'e çıkmış ve grafitteki 2D bandı pik alanının $\sim \% 54$ iken GO sentezlerinde $\sim \% 15$ 'e azalmıştı. D ve G bantlarının şiddet oranı (ID/IG) grafitten grafen okside geçişte, $\sim 0,73$ 'den $\sim 1,00$ 'a yükselmiştir. Bunun yanı sıra D, G ve 2D piklerinin maksimum pik yüksekliklerinin yarıya düştüğü genişlik değerleri sırasıyla; grafitte yaklaşı 42 , 25 ve73 $\mathrm{cm}^{-1}$ iken sentez numunelerinde $\sim 175-187, \sim 79-83$ ve $\sim 300-500 \mathrm{~cm}^{-1}$ aralığında değişmiştir.

Bütün sonuçlar ışığında, bu şartlarda elde edilen sentezlerin, farklı özelliklere sahip grafen oksit örnekleri oldukları ve literatür ile uyum içerisinde oldukları söylenebilir.

Anahtar Kelimeler: Grafit, Grafen oksit, Hummers Yöntemi, Nanomalzeme, Raman Spektroskopisi.

\section{For Graphene Oxide Synthesis Obtained by Modified Hummers Method: Part 4, Raman Spectroscopy Analysis}

\begin{abstract}
In this study, the conversion of graphene to oxide and the change of its structural properties as a result of the syntheses obtained by changing the sodium nitrate concentration in the Hummers method with Raman Spectroscopy analysis were investigated. In the Raman spectrum of graphite, characteristic bands of G (32.3\% peak area) and 2D (54.1\% peak area) were observed at $1573,7 \mathrm{~cm}^{-}$ ${ }^{1}$ and $2692,3 \mathrm{~cm}^{-1}$. In the Raman spectra of graphene oxide syntheses, respectively; two distinct peaks were observed, a $\mathrm{G}$ band $(\sim 23 \%$ peak area) at 1582,1-1591,2 $\mathrm{cm}^{-1}$ and a $\mathrm{D}$ band ( $\sim 51 \%$ peak area) at 1352,3 $-1355,8 \mathrm{~cm}^{-1}$. In addition, a new $\mathrm{D}+\mathrm{D}^{\prime}$ band $(\sim 12 \%$ peak area) appeared in the synthesis samples at $\sim 2928-2947 \mathrm{~cm}^{-1}$ and the $2 \mathrm{D}^{\prime}$ band at $3228 \mathrm{~cm}^{-1}$ in graphite disappeared. After chemical oxidation, in graphene oxide syntheses it increased to $\sim 51 \%$, while the peak area of D band in graphite was $10.8 \%$ and in graphene oxide syntheses it decreased to $\sim 15 \%$ while the peak area of $2 \mathrm{D}$ band in graphite was $\sim 54 \%$. The intensity ratio (ID/IG) of the D and G bands increased from $\sim 0,73$ to $\sim 1,00$ during the transition from graphite to graphene oxide. In addition, the width values at which the maximum peak heights of the $D, G$ and $2 \mathrm{D}$ peaks were varied in the range of $\sim 175-187, \sim 79-83$ and $\sim 300-500 \mathrm{~cm}^{-1}$ in the synthesis samples, while in graphite they were about 42,25 and $73 \mathrm{~cm}^{-1}$, respectively. In the light of all the results, it can be said that the syntheses obtained under these conditions are graphene oxide samples with different properties and are in agreement with the literature.
\end{abstract}




\section{Giriş}

Grafit kristali, altıgen şekilli düzlemlerin birbirlerine çoklu tabakalarla bağlanmış üç boyutlu bir yapı sergiler. Düzlem içindeki karbon atomlarının birbirine güçlü kovalent bağlarıyla, düzlemler arasının ise zayıf Van Der Walls bağlarıyla bağlanmasıyla bu yapı oluşur. Düzlemler arası bu bağların zayıf olmaları sebebiyle tabakalar birbirlerinden çok kolay bir şekilde ayrılırlar. Ayrıca, grafitin düşük maliyeti, kolay elde edilmesi, grafen oksit ile onun türevlerinden oluşan kompozit malzemelere kolaylıkla dönüşebilmesi nedeniyle son yıllarda çok büyük bir ilgi görmektedir [1-2].

Karbon esaslı ve çok tabakalı bir yapıya sahip olan grafitin oksidasyonu sonucu, tabakaların ayrışıp yapıya oksijen içeren fonksiyonel grupların girmesiyle 2 boyutlu grafen oksit (GO) oluşmaktadır. GO'nun yapısındaki bu fonksiyonel grupların karbon atomlarına oranının değişebilmesi, elektronik ve optik özelliklerinin ayarlanabilmesi ve hidrofilik özelliğinden dolayı suda çok iyi disperse olabilmesi nedeniyle; savunma sanayi, enerji, biyokimya, kaplama gibi bir çok teknolojide geniş bir uygulama alanı bulmaktadır. Bunun yanı sıra üretim aşamalarında tehlikeli kimyasalların mevcudiyetinden dolayı bu olumsuzluğu en aza indirebilmek amaciyla son yillarda grafitten grafen oksit eldesi üzerine çalışmalar yapılmaktadır [3-8].

Ayrıca, reaksiyon şartlarıyla GO'nun morfolojik özelliklerinin değișebilmesinden dolayı, sentezlenen her GO'nun literatüre önemli bir katkı sağlayacağı da bildirilmektedir [9].

GO üretim metodlarında kullanılan kimyasal ve oluşan ürünlerin ortak dezavantajları toksik ve tehlikeli olmalarıdır. Bu metodlardan en uygun olanı, üretimin kolaylığı, sentez süresinin kısa oluşu, maliyetin düşük ve yan ürünlerinin diğer yöntemlere göre daha az toksik olmasından dolayı, Hummers yöntemi [10]'dir. Bu yöntemde; oksidan olarak patlayıcı özelliğe sahip $\mathrm{ClO}_{2}$ yerine daha kuvvetli oksitleme özelliği olan $\mathrm{KMnO}_{4}$, dumanlı asit buharı oluşturan nitrik asit yerine sodyum nitrat, grafit ve konsantre karışımı reaksiyona sokulur. Reaksiyonla oluşan $\mathrm{Mn}_{2} \mathrm{O}_{7}$, aromatik yapılar üzerindeki doymamış alifatik çift bağları seçici olarak oksitler[11]. Bu yöntemin dezavantajları ise; sodyum nitrattan dolay1 $\mathrm{NO}_{2}$ ve $\mathrm{N}_{2} \mathrm{O}_{4}$ gibi toksik gazların oluşması, $95{ }^{\circ} \mathrm{C}$ 'nin üzerinde $\mathrm{KMnO}_{4}$ ' 1 n patlama riski ve kristal yapıya giren $\mathrm{Na}^{+}$ve $\mathrm{NO}_{3}{ }^{-}$iyonlarının uzaklaştırılma zorluğudur [12].

$\mathrm{Bu}$ nedenle Hummers yönteminin modifiye edilmesiyle açıklanan dezavantajları azaltabilmek için, reaksiyon şartlarının değiştirilmesi, $\mathrm{KMnO}_{4}$ yerine $\mathrm{K}_{2} \mathrm{FeO}_{4}$ kullanılması, $\mathrm{NaNO}_{3}$ yerine konsantre asit miktarının artırılması veya $\mathrm{H}_{2} \mathrm{SO}_{4}$ ile birlikte $\mathrm{H}_{3} \mathrm{PO}_{4}$ kullanılması gibi çalışmalar yapılmıştır [13].

Raman Spektroskopisi GO'nun karakterizasyonun da çokça kullanılan bir titreşim spektroskopisi olup numune yüzeyine 1 şık kaynağından gönderilen fotonların esnek olmayan saçılmaları incelenerek numune içerisindeki atom veya moleküllerin yapmış olduğu bağlar ve kristal yapıdaki kusurları hakkında bilgi sağlar.

$\mathrm{Bu}$ çalışmada, Raman Spektroskopisi analizi ile değişik $\mathrm{NaNO}_{3}$ konsantrasyonlarında elde edilen sentezlerin grafen oksite dönüşümü ve değişen yapısal özelliklerinin incelenmesi amaçlanmıştır.

\section{Materyal ve Metot}

\subsection{Kullanılan Kimyasallar ve Materyaller}

Çalışma kimyasalları olarak; merck-325 meş grafit tozu, mercksodyum nitrat, merck-\%98'lik sülfürik asit, merck-potasyum permanganat, ultra deiyonize su, merck-\%30'luk hidrojen peroksit ve fluka-\%37'lik hidroklorik asit kullanılmıştır. Materyaller ise; $250 \mathrm{ml}$ 'lik cam reaktör, ultrasonik banyo $150 \mathrm{w}$ (çalışkan lab.), çeker ocak, 1sıtıcılı manyetik karıştırıcı (1ka yellowline msh basic), $\mathrm{pH}$ metre (mettler toledo), santifürüj cihazı, etüv, termometre şeklindedir.

\subsection{Deneysel Sistem}

Grafen oksit sentezlerinin deneysel akış şeması Şekil 1'de verilmiştir. Reaksiyonun gerçekleştiği $250 \mathrm{~mL}$ 'lik cam reaktör içerisine, $1 \mathrm{~g}$ grafit tozu, $0-1 \mathrm{~g}$ aralığında değişen $\mathrm{NaNO}_{3}$ ve 23 $\mathrm{mL} \mathrm{H}_{2} \mathrm{SO}_{4}$ ilave edilmiştir. Karışım $30 \mathrm{dk}$ karıştırılıp literatürdeki diğer modifiye Hummers yöntemlerinden farklı olarak 40 dakika boyunca $-15{ }^{\circ} \mathrm{C}$ 'de dondurucuda bekletilmiştir. Karıştırma hızı, homojen bir süspansiyonun elde edildiği 500 rpm'e ayarlanmıştır. Dondurucudaki numune buz banyosuna konularak $0^{\circ} \mathrm{C}$ 'ye getirilmiş ve yaklaşık 1 saat boyunca kademeli olarak $3 \mathrm{~g} \mathrm{KMnO}_{4}$ ilave edilerek Eşitlik 1-2'deki oksidasyon reaksiyonlarıyla koyu yeşil renkli grafit oksit oluşumu gözlenmiştir (bu aşamada sıcaklık $5^{\circ} \mathrm{C}^{\prime}$ yi geçmemesine dikkat edilmelidir) [14].

$\mathrm{KMnO}_{4}+3 \mathrm{H}_{2} \mathrm{SO}_{4} \rightarrow K^{+}+\mathrm{MnO}_{3}^{+}+\mathrm{H}_{3} \mathrm{O}+3 \mathrm{HSO}_{4}^{-}$

$\mathrm{MnO}_{3}^{+}+\mathrm{MnO}_{4}^{-} \rightarrow \mathrm{Mn}_{2} \mathrm{O}_{7}$

Karışımı mekanik olarak GO'ya dönüştürebilmek için, $35^{\circ} \mathrm{C}$ sıcaklıkta 2 saat 500 rpm'de karıştırılmıştır (sıcaklık bu aşamada $50{ }^{\circ} \mathrm{C}$ 'yi geçmemesine dikkat edilmelidir). Süre sonunda açık kahverenkli karışıma $98^{\circ} \mathrm{C}$ sıcaklıkta kontrollü bir biçimde $46 \mathrm{~mL}$ ultra saf su ilave edilmiş ve $15 \mathrm{dk}$ daha karıştırılmıştır (Bu esnada rengin koyu kahveye dönüştüğü gözlenmiştir). Daha sonra, 140 $\mathrm{mL}$ ultra saf su ve $8 \mathrm{~mL} \mathrm{H}_{2} \mathrm{O}_{2}$ ilavesiyle renk sarıya dönmüş ve $\mathrm{KMnO}_{4}$ fazlası Eşitlik (3)'de gösterildiği gibi suda çözünen mangan sülfat tuzuna dönüştürülmüştür.

$2 \mathrm{KMnO}_{4}+3 \mathrm{H}_{2} \mathrm{SO}_{4}+3 \mathrm{H}_{2} \mathrm{O}_{2} \rightarrow 2 \mathrm{MnSO}_{4}+2 \mathrm{~K}_{2} \mathrm{SO}_{4}+8 \mathrm{H}_{2} \mathrm{O}+$ $5 \mathrm{O}_{2}$

Son karışım 24 saat oda sıcaklığında bekletilmiş ve süzülerek ayrilan katı numune, \%10'luk $\mathrm{HCl}$ ile bünyesindeki metal iyonlarını gidermek için ve saf su ile reaksiyona girmeyen asit fazlasını gidermek için pH 7 olana kadar yıkanmıştır. Karışım 60 ${ }^{\circ} \mathrm{C}$ 'ye ayarlanmış etüvde 4 saat kurutulmuş sonra saf su ilave edilerek elde edilen GO süspansiyonu 2 saat sonikasyona (güç: $150 \mathrm{~W}$ ve frekans: $33 \mathrm{KHz}$ ) tabi tutulmuş ve daha sonra $5 \mathrm{dk}$ 
boyunca 3000 dev.dk ${ }^{-1}$ hız ile santifürüj edilerek çözeltisinden ayrılmıştır. Son olarak, katı numune $60{ }^{\circ} \mathrm{C}$ 'deki etüvde 24 saat kurutulmuş ve GO sentezi gerçekleştirilmiştir.

Elde edilen örneklere $\mathrm{NaNO}_{3}{ }^{\prime}$ 'n artan konsantrasyonuna göre sırasıyla, GO-Na0 ve GO-Na0,50 kodları verilmiştir.

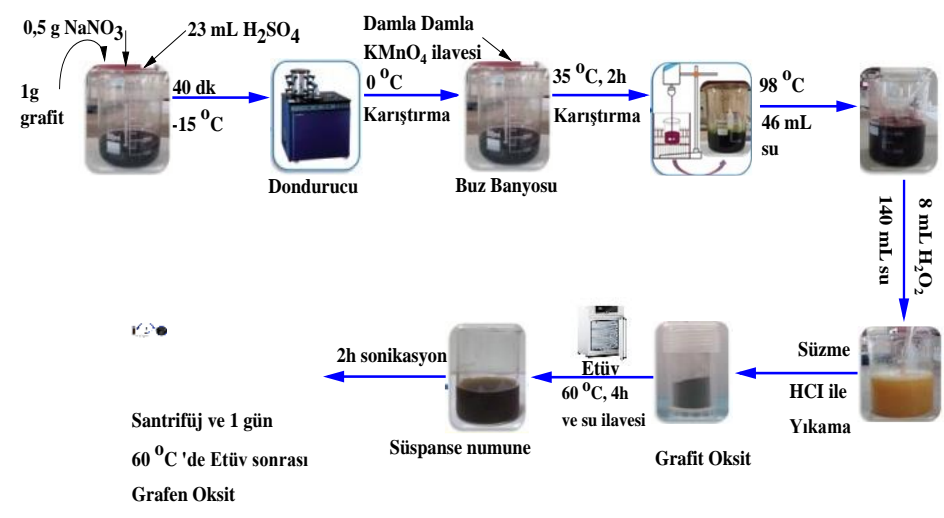

Şekil 1. Grafen Oksit Sentezi için Deneysel Akış Şeması

\subsection{Raman Spektroskopisi Analizi}

Raman spektroskopisi analizi ile, numune içerisindeki atom veya moleküllerin yapmış olduğu bağlar ve kristal yapıdaki kusurları hakkında bilgi sağlandığından dolayı grafen türevli malzemelerin molekül yapısını aydınlatılabilen çok iyi bir karakterizasyon aracıdır.

GO'nun Raman spektrumlarında; D, G ve 2D bandlarındaki piklerin şiddet oranları ile maksimum pik yüksekliklerinin yarıya düştüğü genişlik (FWHM) değerleri, molekül yapısının aydınlatılmasına katkı sağlayan en önemli bilgilerdir. Bunun yanı sıra D+D' ve 2D' bandlarındaki pikler de katkı sağlamaktadır [15].

Bu çalışmada WITech alpha 300R model spektroskopisi kullanılmıştır.

\section{Araştırma Sonuçları ve Tartışma}

Grafit ve seçilmiş GO sentezlerinin Raman spektrumları Şekil 2-3'de grafik edilmiş ve grafiklerden okunan değerler Tablo 1 'de sunulmuştur. Tablo 1'deki sinyal veren bantların dalga sayıları, FWHM ve pik alanı değerleri; Gaussian (Gsn, R²:0,972) ve Lorentzian ( $\left.\mathrm{Lrz}, \mathrm{R}^{2}: 0,988\right)$ dağılım analizlerine göre ayrı ayrı belirlenmiştir.

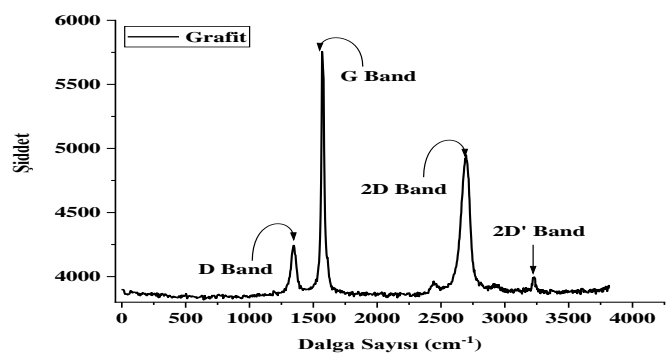

Şekil 2. Grafitin Raman Spektrumu

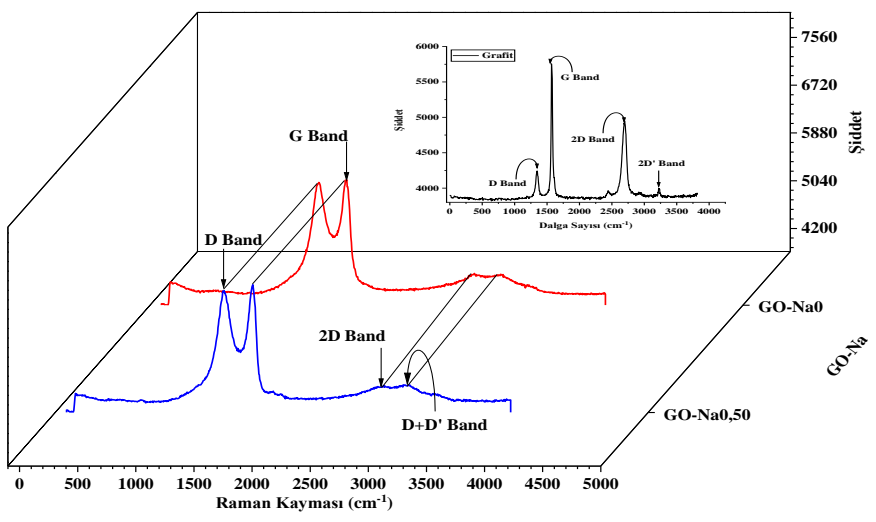

Şekil 3. GO-Na Raman Spektrumu

Tablo 1. Grafit ve Seçilen GO Sentezlerinin Raman Spektroskopisi Analiz Sonuçları

\begin{tabular}{|c|c|c|c|c|}
\hline \multicolumn{2}{|l|}{ Parametre } & Grafit & GO-Na0 & GO-Na0,50 \\
\hline \multicolumn{2}{|l|}{$\mathbf{I}_{\mathrm{D}} / \mathbf{I}_{\mathrm{G}}$} & 0,73 & 0,993 & 0,983 \\
\hline \multirow{2}{*}{$\begin{array}{l}\text { D Bandı } \\
\left(\mathrm{cm}^{-1}\right)\end{array}$} & Gsn & 1345,7 & 1357,2 & 1360,6 \\
\hline & Lrz & 1345,6 & 1352,3 & 1355,8 \\
\hline \multicolumn{2}{|l|}{ D Bandı Siddeti } & 4243 & 5955 & 5937 \\
\hline \multirow[t]{2}{*}{ G Bandı $\left(\mathrm{cm}^{-1}\right)$} & Gsn & 1573,8 & 1583,0 & 1589,5 \\
\hline & Lrz & 1573,7 & 1582,1 & 1591,2 \\
\hline \multicolumn{2}{|l|}{ G Bandı Şiddeti } & 5756 & 5998 & 6042 \\
\hline \multirow[t]{2}{*}{ 2D Bandı $\left(\mathrm{cm}^{-1}\right)$} & Gsn & 2691,5 & 2681,0 & 2711,6 \\
\hline & Lrz & 2692,3 & 2668,6 & 2687,5 \\
\hline \multicolumn{2}{|l|}{ 2D Bandı Şiddeti } & 4353 & 4263 & 4240 \\
\hline \multirow{2}{*}{$\begin{array}{l}\text { D+D' Bandı } \\
\left(\mathrm{cm}^{-1}\right)\end{array}$} & Gsn & - & 2946,5 & 2961,9 \\
\hline & Lrz & - & 2927,4 & 2946,7 \\
\hline \multirow{2}{*}{$\begin{array}{l}\text { 2D' Bandı } \\
\left(\mathrm{cm}^{-1}\right)\end{array}$} & Gsn & 3228,0 & - & - \\
\hline & Lrz & 3228,0 & - & - \\
\hline \multirow{2}{*}{$\begin{array}{l}\text { FWHM } \\
\left(\mathrm{D}, \mathrm{cm}^{-1}\right)\end{array}$} & Gsn & 50,27 & 226,1 & 213,1 \\
\hline & Lrz & 41,75 & 187,3 & 175,1 \\
\hline \multirow{2}{*}{$\begin{array}{l}\text { FWHM } \\
\left(\mathrm{G}, \mathrm{cm}^{-1}\right)\end{array}$} & Gsn & 29,79 & 101,3 & 96,6 \\
\hline & Lrz & 24,71 & 82,8 & 78,6 \\
\hline \multirow{2}{*}{$\begin{array}{l}\text { FWHM } \\
\left(2 \mathrm{D}, \mathrm{cm}^{-1}\right)\end{array}$} & Gsn & 85,59 & 325,2 & 475,8 \\
\hline & Lrz & 72,94 & 300,5 & 552,9 \\
\hline \multirow[t]{2}{*}{ D Pik alanı (\%) } & Gsn & 10,7 & 56,0 & 54,1 \\
\hline & Lrz & 10,8 & 52,8 & 49,6 \\
\hline \multirow[t]{2}{*}{ G Pik alanı (\%) } & Gsn & 33,1 & 26,1 & 26,9 \\
\hline & Lrz & 32,3 & 22,8 & 22,8 \\
\hline \multirow[t]{2}{*}{ 2D Pik alanı (\%) } & Gsn & 54,2 & 9,4 & 13,8 \\
\hline & Lrz & 54,1 & 9,3 & 19,3 \\
\hline \multirow[t]{2}{*}{ 2D' Pik alanı (\%) } & Gsn & 2,0 & - & - \\
\hline & Lrz & 2,8 & - & - \\
\hline \multirow[t]{2}{*}{ D+D' Pik alanı (\%) } & Gsn & - & 8,5 & 5,1 \\
\hline & Lrz & - & 15,1 & 8,4 \\
\hline
\end{tabular}




\section{Sonuç}

Şekil 2-3 ve Tablo 1 incelenildiğinde aşağıdaki sonuçlar elde edilmiştir.

Grafitte, iki güçlü bant gözlemlenmiş olup dalga sayısı Lorentzian dağılımına göre bunlar, $1573,7 \mathrm{~cm}^{-1}$ de $\mathrm{G}(\% 32,3$ pik alanl1) ve $2692,3 \mathrm{~cm}^{-1}$ de 2D (\%54,1 pik alanlı) bantlarıdır. G bandı, hem halkalı hem de düz zincirli yapılardaki $\mathrm{sp}^{2}$ karbon atomlarının bağ gerilmesi nedeniyle oluşan birinci dereceden spektrumdur. 2D bandının şiddeti ve genişliğinin fazla olması ise, malzemelerin çok tabakadan oluştuğunun göstergesidir [16]. Grafitte ayrıca, D (\%10,8 pik alanlı) ve 2D' (\%2,8 pik alanlı) bantları da sırasıyla 1345,6 ve $3228 \quad \mathrm{~cm}^{-1}$ de oluştuğu gözlemlenmiştir. Bu bandların mevcudiyeti, grafitteki boşluklar ve amorf karbon türleri gibi bazı yapısal bozukluğun bir göstergesidir [17-18].

GO-Na0 ve GO-Na0,50 sentezlerinin Raman spektrumlarında sirasiyla; 1582,1 ve $1591,2 \mathrm{~cm}^{-1}$, de $\mathrm{G}$ bandı ( \%23 pik alanlı) ve 1352,3 ve $1355,8 \mathrm{~cm}^{-1}$ de bir D band1 ( $\% 51$ pik alanlı) olmak üzere iki belirgin pik gözlenmiş̧ir. Buradaki bant değerlerinin grafitteki bant değerlerine göre kaymalarının sebebi, karbon tabakalarında oluşan fonksiyonel gruplara ve GO$\mathrm{Na}$ sentezlerindeki düşük band kaymalarının sebebi ise $\mathrm{NaNO}_{3}$ konsantrasyonu değerinin değişmesine atfedilebilir [19].

Kimyasal oksidasyondan sonra, grafitteki D band1 pik alanı \%10.8 iken, GO sentezlerinde $\% 51$ 'e yükselmesi sonrası ve grafitteki $2 \mathrm{D}$ bandı pik alanı $\% 54$ iken, GO sentezlerinde \%15'e azalması, bu sentezlerin başarılı bir şekilde gerçekleştiğini ve literatür değerleri ile uyum içerisinde olduğunu göstermiştir [20]. 2D bandının yoğunluğundaki etkin azalma ise, oksidasyon reaksiyonu sonucu tabakaların kırılmasına atfedilir [21]. Buna ilaveten GO-Na sentezlerinde $2928-2947 \mathrm{~cm}^{-1}$ de kusur oluşumunu doğrulayan yeni bir D+D' bandı ( \%12 pik alanlı) ortaya çıkmış ve grafitte $3228 \mathrm{~cm}^{-1}$ deki 2D' bandı ise kaybolmuştur. D+D' bandının oluşumu, GO örneklerini grafitten ayıran önemli bir kanıttır [22]. Grafit ile karşılaş̧ırıldığında, GO$\mathrm{Na}$ örneklerindeki zayıf ve geniş 2D piki ile birlikte belirgin olan $\mathrm{D}$ piki, grafit yapısındaki hibridize $\mathrm{sp}^{2} \mathrm{C}$ atomlarının yapısının bozulması sonucu GO'ya dönüştürüldüğünün ana belirtileridir.

$D$ ve $G$ bantlarının şiddet oranı (ID/IG), grafit tabakalarındaki bozulma derecesinin iyi bir göstergesidir. Grafitten grafen okside geçişte ID/IG oranının $\sim 0,73$ 'den $\sim 1,00$ 'a artmas1; $\mathrm{sp}^{2}$ bağlı karbon atomlarının büyük kısmının $\mathrm{sp}^{3}$ bağlı karbon atomlarına dönüştürülmesiyle düzlem içi kristalde bir azalmayı ve grafitin kısmi olarak amorf yapı göstermesi, oksidasyonun başarısını destekler.

ID/IG'nin yanısıra $\mathrm{D}, \mathrm{G}$ ve $2 \mathrm{D}$ piklerinin FWHM değerleri de oksidasyonun başarısının bir göstergesidir. Lorentzian dağılımına göre $\mathrm{D}, \mathrm{G}$ ve $2 \mathrm{D}$ bantlarının FWHM değerleri sırasıyla grafitte yaklaşık 42,25 ve $73 \mathrm{~cm}^{-1}$ iken GO-Na sentezlerinde $~ 175-187$, $\sim 79-83$ ve $\sim 300-500 \mathrm{~cm}^{-1}$ aralığında değişmektedir. G pikinin FWHM'sinin küçük olmasının nedeni, grafen oksit tabakalarındaki $\mathrm{sp}^{2}$ hibridize karbon bağlarının gerilme titreşimlerinden kaynaklanır. D pikinin FWHM'sinin büyük olması ise yapıdaki hetero atomlardan dolayı oluşan yapısal bozukluk anlamına gelir [17].

Bütün sonuçlar ışığında, bu şartlarda elde edilen sentezlerin, farklı özelliklere sahip grafen oksit örnekleri oldukları ve literatür ile uyum içerisinde oldukları söylenebilir.

\section{Teşekkür}

Bu çalışma, Atatürk Üniversitesi BAPSİS Birimi tarafından Temel Araştırma Projesi olarak desteklenmiştir.

\section{Kaynakça}

[1] Moosa, A., and Abed, M. (2021). Graphene preparation and grapfite exfoliation. Turkish journal of Chemistry, 45(3), 493-519.

[2] Dresselhaus, G., Dresselhaus, M. S., \& Saito, R. (1998). Physical properties of carbon nanotubes. World scientific.

[3] Tiyek, İ., Dönmez, U., Yıldırım, B., Alma, M. H., Ersoy, M. S., \& Karataş, Ş. (2016). Kimyasal yöntem ile indirgenmiş grafen oksit sentezi ve karakterizasyonu. Sakarya University Journal of Science, 20(2), 349-357.

[4] Paulchamy, B., Arthi, G., \& Lignesh, B. D. (2015). A simple approach to stepwise synthesis of graphene oxide nanomaterial. J Nanomed Nanotechnol, 6(1), 1.

[5] Brisebois, P. P., \& Siaj, M. (2020). Harvesting graphene oxide-years 1859 to 2019: a review of its structure, synthesis, properties and exfoliation. Journal of Materials Chemistry C, 8(5), 1517-1547.

[6] Sun, L., \& Fugetsu, B. (2013). Mass production of graphene oxide from expanded graphite. Materials Letters, 109, $207-$ 210.

[7] Huang, X., Qi, X., Boey, F., \& Zhang, H. (2012). Graphenebased composites. Chemical Society Reviews, 41(2), 666686.

[8] Chen, J., Yao, B., Li, C., \& Shi, G. (2013). An improved Hummers method for eco-friendly synthesis of graphene oxide. Carbon, 64, 225-229.

[9] Shamaila, S., Sajjad, A. K. L., \& Iqbal, A. (2016). Modifications in development of graphene oxide synthetic routes. Chemical Engineering Journal, 294, 458-477.

[10] Hummers Jr, W. S., \& Offeman, R. E. (1958). Preparation of graphitic oxide. Journal of the american chemical society, 80(6), 1339-1339.

[11] Dreyer, D. R., Park, S., Bielawski, C. W., \& Ruoff, R. S. (2010). The chemistry of graphene oxide. Chemical society reviews, 39(1), 228-240.

[12] Lavin-Lopez, M. D. P., Romero, A., Garrido, J., SanchezSilva, L., \& Valverde, J. L. (2016). Influence of different improved hummers method modifications on the characteristics of graphite oxide in order to make a more easily scalable method. Industrial \& Engineering Chemistry Research, 55(50), 12836-12847.

[13] Marcano, D. C., Kosynkin, D. V., Berlin, J. M., Sinitskii, A., Sun, Z., Slesarev, A., ... \& Tour, J. M. (2010). Improved synthesis of graphene oxide. ACS nano, 4(8), 4806-4814. 
[14] Peng, L., Xu, Z., Liu, Z., Wei, Y., Sun, H., Li, Z., ... \& Gao, C. (2015). An iron-based green approach to 1-h production of single-layer graphene oxide. Nature communications, 6(1), 1-9.

[15] Ferrari, A. C., \& Basko, D. M. (2013). Raman spectroscopy as a versatile tool for studying the properties of graphene. Nature nanotechnology, 8(4), 235-246.

[16] Gupta, A., Chen, G., Joshi, P., Tadigadapa, S., \& Eklund, P. C. (2006). Raman scattering from high-frequency phonons in supported n-graphene layer films. Nano letters, 6(12), 2667-2673.

[17] Ferrari, A. C., \& Robertson, J. (2000). Interpretation of Raman spectra of disordered and amorphous carbon. Physical review B, 61(20), 14095.

[18] Schönfelder, R., Rümmeli, M. H., Gruner, W., Löffler, M., Acker, J., Hoffmann, V., ... \& Pichler, T. (2007). Purification-induced sidewall functionalization of magnetically pure single-walled carbon nanotubes. Nanotechnology, 18(37), 375601.

[19] Bokobza, L., Bruneel, J. L., \& Couzi, M. (2015). Raman spectra of carbon-based materials (from graphite to carbon black) and of some silicone composites. C-Journal of Carbon Research, 1(1), 77-94.

[20] Tuinstra, F., \& Koenig, J. L. (1970). Raman spectrum of graphite. The Journal of chemical physics, 53(3), 11261130.

[21] Krishnamoorthy, K., Veerapandian, M., Yun, K., \& Kim, S. J. (2013). The chemical and structural analysis of graphene oxide with different degrees of oxidation. Carbon, 53, 3849.

[22] Aliyev, E., Filiz, V., Khan, M. M., Lee, Y. J., Abetz, C., \& Abetz, V. (2019). Structural characterization of graphene oxide: Surface functional groups and fractionated oxidative debris. Nanomaterials, 9(8), 1180. 